\title{
Application of Topology Optimization to Thighbone/Implant Interaction Modelling
}

\author{
Ryszard Kutyłowski ${ }^{1 *}$, Marek Szwechłowicz' \\ ${ }^{1}$ Faculty of Civil Engineering, Wrocław University of Science and Technology, Wybrzeże St. Wyspiańskiego 27, 50-370 Wrocław, \\ Poland \\ * Corresponding author, e-mail: ryszard.kutylowski@pwr.edu.pl
}

Received: 30 January 2020, Accepted: 19 April 2020, Published online: 28 May 2020

\begin{abstract}
This paper presents an application of topology optimization in the analysis of the thighbone with an inserted endoprosthesis. A variational minimum compliance approach with mass constraints is employed to solve the problem. Changes in the bone structure depending on the implant's mechanical parameters are studied. Numerical results for mass distribution in the modelled thighbone with an inserted endoprosthesis are analyzed using an original numerical algorithm and a program developed in Matlab. It is numerically proven that owing to the use of an implant with material properties similar to those of the bone no degradation of the latter in the vicinity of the implant occurs. The endoprosthesis will serve longer and the postoperative complications connected with the accelerated degradation of the bone in the neighborhood of the implant will be avoided if the implant has similar material properties as the bone.
\end{abstract}

Keywords

topology optimization, numerical modelling of the thighbone with an endoprosthesis, endoprosthesis material properties, bone degradation

\section{Introduction}

In this paper the interaction between the implant and the bone, i.e. the distribution of material when an endoprosthesis modelling structure is introduced into the design domain, was numerically analyzed.

The theoretical basis of the approach used here, and the authors' numerical algorithm and program written in Matlab together with a practical civil engineering implementation were presented in [1]. The optimization problem there was formulated variationally. The compliance functional minimalization approach under constraints imposed on the body mass available for optimization was adopted. The problem of the optimal distribution of the available material in a strictly defined design area under the assumed boundary conditions and the prescribed load was considered.

Global constraints were imposed on body mass in $[1,2]$ and in the present paper. This means that depending on the amount of available mass, one gets different optimal distributions of this mass in the design area, whereby having more mass one gets a structure whose elements fill up the design area more tightly and this structure is stiffer than the one obtained for a smaller amount of mass. Considering the problem of bone tissue distribution around the implant from the physical point of view, the imposition of constraints on body mass should be understood as follows: a relatively large body mass is available in the case of a person with healthy bone tissue, whereas a small body mass occurs in a person with considerably advanced osteoporosis. For example, when a small mass is available, the question is how to distribute this little mass in order to obtain the maximum stiffness of the bone structure.

The numerical modelling of a thighbone structure by means of the same algorithm and program was presented in [2]. The structure was loaded by representative force $W$ or $P$ (Fig. 1) to reveal the general tendency regarding the considered problems. From the practical point of view, research on the implant/bone interaction is highly important as it aims to minimize the adverse effect of the implant on the bone structure. The topic is discussed in, e.g., [3] where it is proposed to use functionally gradient materials (FGM) for producing implants to avoid problems arising at the contact between the two materials (bone/implant) characterized by different physical properties, and bone 
resorption problems. The latest achievements in bone/ implant interactions, including coated porous implant/ bone interactions, are discussed in [4]. Various methods are considered to enhance the biocompatibility of the materials used during the operation. For example, in [5] the influence of nano-hydroxyapatite (HA) on the fracture behavior of acrylic bone cement (PMMA) under tension-shear loading was investigated.

Since generally there is a biomechanical mismatch between the implant and the surrounding tissues, mainly due to the different Young moduli of the two, intensive research on the potential use of materials with a gradient structure is underway in bioengineering. The present study is based on [6] where the degradation of the bone in the vicinity of the implant was demonstrated numerically. This is confirmed here, and it is shown how to avoid such degradation. Current achievements in the modelling of new-generation implants are presented in [7], which includes a discussion of ways in which post-implantation degradation can be minimized. Initially, materials such as stainless steel, pure titanium, or its alloys, which are characterized by much higher Young moduli than compact bone, were used in implantology. Today new-generation materials begin to be used. It should be noted that there is ongoing research aimed at finding a material which would interact with the natural bone structure. An example here it the research conducted at the Medical University in Lublin (Poland), where a bone replacing composite material characterized by high biocompatibility was developed and patented [8]. It is worth mentioning that in [9] a novel cementless stem characterized by a gradual decrease in Young's modulus was proposed. Numerical simulations showed that 10 years after its implantation the bone mineral density would be over $40 \%$ higher than for other implants.

A comprehensive review of the problem of bone degeneration around the implant is presented in [14], where a definition of the problem is given. It is also noted there that as a result of implantation, stresses in the bone decrease and the atrophy of the bone around the implant is observed. Also, a procedure for reducing bone atrophy is demonstrated.

Another interesting review-like study is [15], where it is mentioned that professor Huiskes was the first to draw attention to this problem and link it with the stress strain state in the bone. Also, the stress-shielding problem and its practical solution are extensively discussed in [15]. It is mentioned that animal testing demonstrated that when less stiff implants were used, the bones degraded to a lesser degree.
All the above studies show that this problem exists, but none of it proves that from the point of view of the laws of physics the degradation of the bone around the implant is a natural phenomenon. The human skeletal system is being built and remodeled in the course of life, also after implantation, and is optimally constructed. Therefor it follows from the principles of optimal bone structure design that the bone around the implant often undergoes degeneration. This will be proven numerically, on the basis of the theory presented in $[1,2]$, in the next sections.

\section{Thighbone/implant interaction 2.1 Implant modelling}

This paper continues the analysis presented in [2]. In this part of the research the changes in the structure of the thighbone after the introduction of an implant made of a uniformly dense material were studied in order to determine the effect of the hip joint implant on the structure of the thighbone.

In the case of a healthy thighbone, a load applied to its upper part is carried by the spongy bone trabeculae and then transferred via cortical bone to the thighbone's lower parts.

When an implant is inserted into the thighbone, the way in which load is transferred to its lower parts changes since load is then applied to the implant head and most of the load via the implant is transferred directly to the cortical bone and then through the latter to the lower parts of the thighbone. The rest (a very small part) of the load is transferred through the spongy bone in the thighbone's upper part to the cortical bone. Since the implant is very stiff in comparison with the bone, nearly all the load is carried by the implant whereby the bone in the vicinity of the implant is not sufficiently loaded, to which the body reacts by increasing the activity of osteoclasts. The overactivity of the osteoclasts contributes to quick resorption (loss) of bone tissue, leading to changes in the bone structure. Fig. 1(a) shows an exemplary endoprosthesis of the hip joint. The finite elements making up the hip joint endoprosthesis in computations are marked in Fig. 1(b).

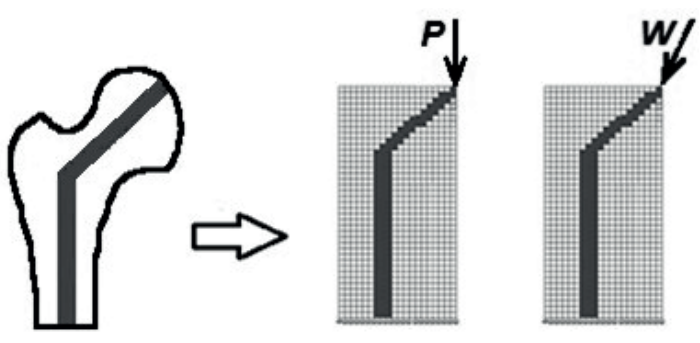

Fig. 1 Hip joint endoprosthesis (a) and computational model (b) 
Considering that due to the weakening of the bone structure in the vicinity of, particularly, the implant shaft the prosthesis often becomes loose, the main focus in the computations was on the effect of the implant shaft on the thigh bone. In the analyzed cases, the implant was inserted into the core of the thighbone and loaded with concentrated force $P$ or $W-$ similar as it was in [2]. These forces are representative forces for the considered problem.

It appears from the topologies presented in this section that bone density significantly changes as a result of implantation. Due to the fact, that the load is carried mainly by the implant the bone tissue in the vicinity of the implant undergoes degradation. Today an ever deeper insight into the processes of repeated bone remodeling (commensurate with the stress strain state of the bone in the preceding period) during a person's life is being gained [10]. As a result, the degradation of the bone tissue in the neighborhood of the implant, leading to the loosening of the implant, is better understood [11]. In recent years some sophisticated research in this field has been done. For example, 3D tissue scaffolds with a controlled internal architecture are presented in [12] and human trabecular bone core scanning and advanced FEM intrinsic material modelling are reported in [13].

\subsection{Solutions for two implant cases}

Studies showing how the bone structure remodels at the implant/bone contact are presented below. First an example similar to the one presented in Fig. 15 in [2], but for a greater amount of mass, is shown. When in the course of optimization one increases the amount of mass available for bone tissue building (by increasing mass reduction coefficient $\alpha$ from 0.3 to 0.42 (Fig. 2) or to 0.5 (Fig. 3)), this has no significant effect on the shape of the obtained topologies. In the examples, 384 elements were allocated for implant building, which at assumed available mass $m_{0}=1344$ amounts to $28 \%$ (for $\alpha=0.42$ ) while at $m_{0}=1600$ it amounts to $24 \%$ (for $\alpha=0.50$ ).

Fig. 2(a) presents the final material/void (1/0) solution for the implant shown in Fig. 1. Fig. 2(b) shows a normalized deformation energy distribution on a density scale of 0-1 (only the implant is marked black). In order to better visualize the actual variation in material density distribution the topology was examined on different density scales: 0-0.1 (Fig. 2(c)), 0-0.02 (Fig. 2(d)), 0-0.015 (Fig. 2(e)) and 0-0.001 (Fig. 2(f)). This means that, for example, the upper limit as equal not to 1 , but to 0.1 . Consequently, the interval of 0-0.1 is divided into 10 shades of grey, as shown in

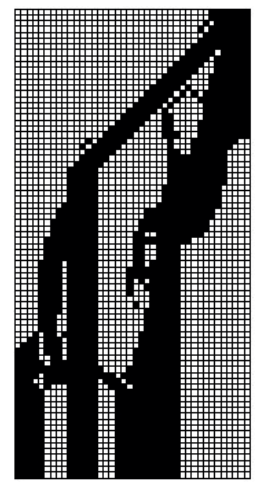

(a)

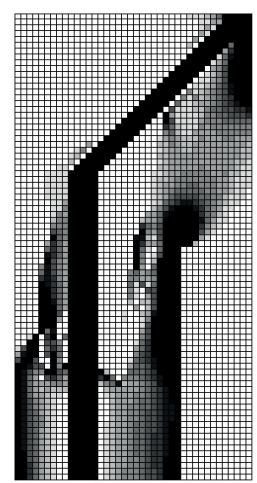

(d)

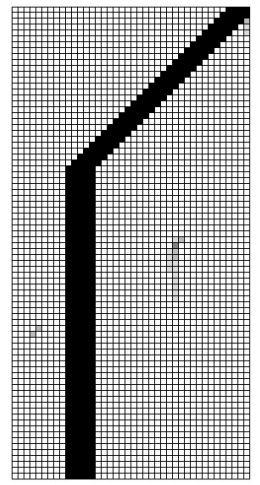

(b)

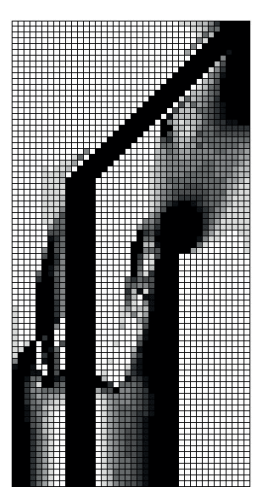

(e)

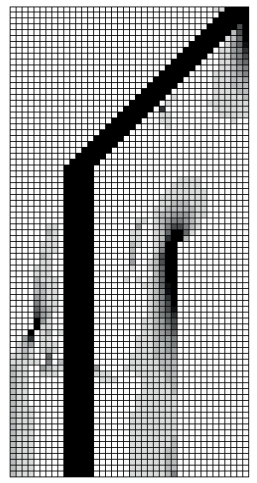

(c)

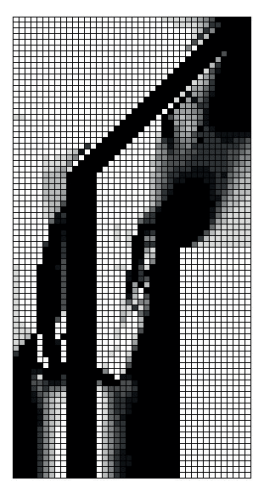

(f)
Fig. 2 Topologies of density distribution in step 10 under loading with force $P$ for $\alpha=0.42$; 0-1 (a); 0-1 (b); 0-0.1 (c); 0-0.02 (d); 0-0.015 (e); $0-0.001$ (f)

Fig. 2(c), where, e.g., the white color corresponds to the numbers from the interval of 0-0.01 while the black color corresponds to the numbers from 0.09 to 1 inclusive.

Fig. 3 shows, in the same configuration as previously, topologies for $\alpha=0.50$. Also in this case it was found that the introduced implant contributed to the remodeling of the bone structure and to the atrophy of certain elements situated above the slanting part of the prosthesis and on the left side. In addition, in this distribution (Fig. 3) the horizontal bars in the bone marrow core, visible in Fig. 2, have disappeared and in their place a bar originating at an angle from the right side towards the thighbone head has formed.

The topologies shown in Fig. $2(\alpha=0.42)$ and Fig. 3 $(\alpha=0.50)$ confirm the earlier observations [2] that implantation contributes to the considerable remodeling of the bone structure. As a result, the areas above the slanting part of the prosthesis (the thighbone head) undergo weakening. Also, the cortical bone on the left side is considerably weakened. More material is located on the right side, closer to the force application point. After implantation the structure of the bone changes. Additional horizontal 


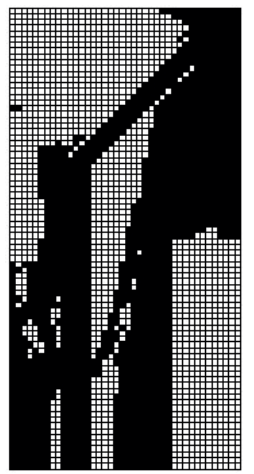

(a)

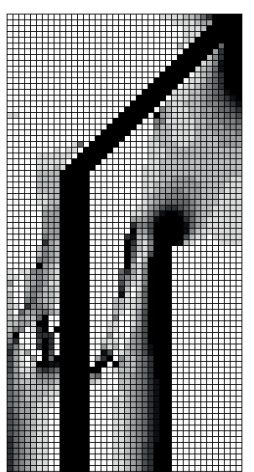

(d)

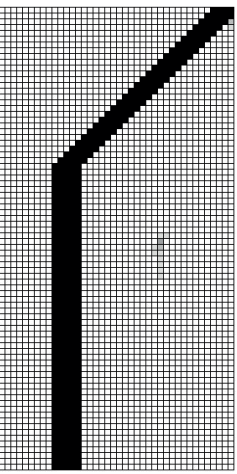

(b)

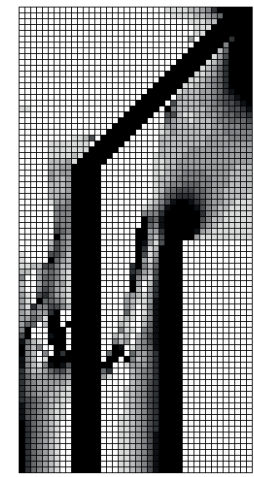

(e)

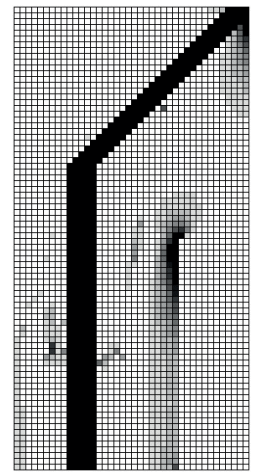

(c)

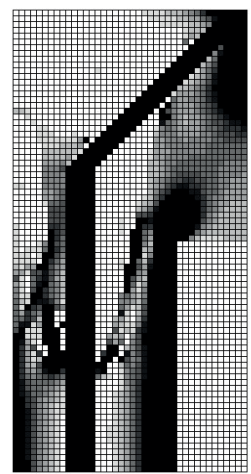

(f)
Fig. 3 Topologies of density distribution in step 4 under loading with force $P$ for $\alpha=0.5$ - 0-1 (a); 0-1 (b); 0-0.1 (c); 0-0.02 (d); 0-0.015 (e); $0-0.001$ (f)

connections, in the form of single bars originating from the implant and extending to the right and to the left towards the cortical bone (Figs. 2(d)-(f)), develop.

To see how the solutions are changing when healthy bone solutions are compared with the ones obtained for the bone with the implant one should consider the topologies with the same available mass allocated, used for building the bone alone (without the mass allocated to the implant). And so, for example, the solution shown in Fig. 6(c) in [2] should be compared with the one in Fig. 3(a) $(\alpha=0.50)$. Fig. 4 shows a 0/1 distribution topology without the implant (Fig. 4(a)) and with the implant (Fig. 4(b)), where the amounts of available mass used to build the bone alone are the same.

In Fig. 4(b) the implant which (for the $40 \times 80$ grid) comprises 384 elements with a density of 1 is shown in the same shape as in Fig. 1(b). One can see how the distribution of the same amount of mass changes when the implant takes over almost the whole load.

Then a case in which the amount of material for the implant was reduced, with the amount by which it was reduced allocated for bone building, was analyzed. The implant in this case became "thinner". Figs. 5 and 6 show the results of computations for the loading with force $P$.

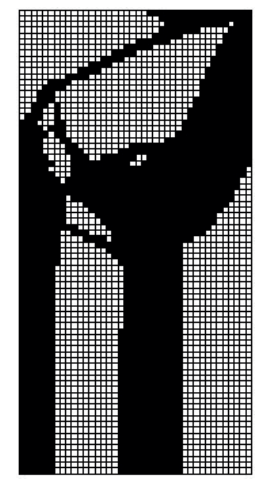

(a)

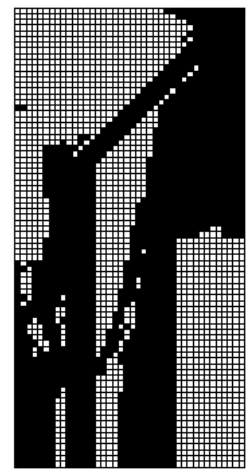

(b)
Fig. 4 0/1 topologies under loading with force $P$, without implant from Fig. 6(c) [2] (a); and with implant from Fig. 3(a) (b)

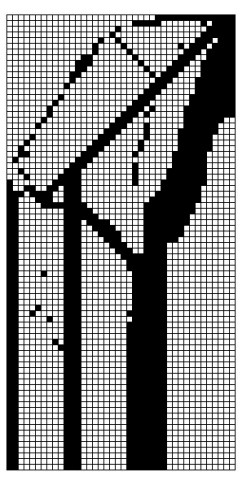

(a)

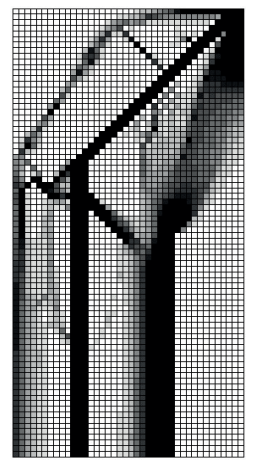

(d)

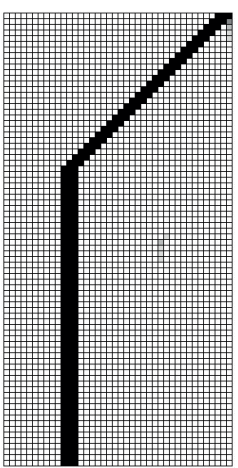

(b)

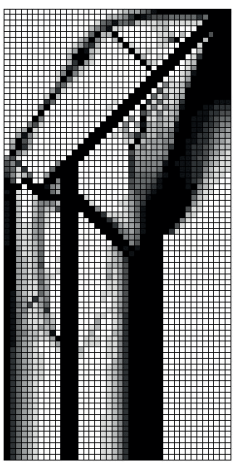

(e)

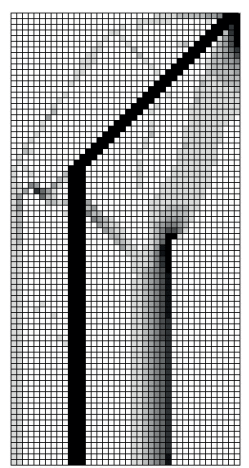

(c)

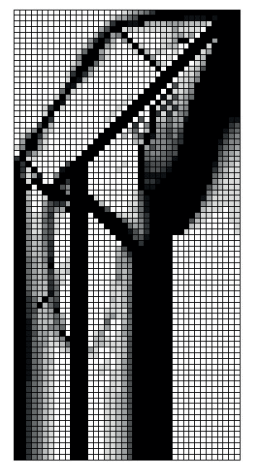

(f)
Fig. 5 Topologies of density distribution in step 19 under loading with force $P$ ("thin" implant) for $\alpha=0.30 ; 0-1$ (a); 0-1 (b); 0-0.1 (c); 0-0.02

$$
\text { (d); 0-0.015 (e); 0-0.001 (f) }
$$

When the amount of material for bone structure building was reduced at the expense of the amount for implant building, it was found that the implant no longer carried the whole load but shared it with the thighbone.

In this solution (Figs. 5(d), (e), (f)) the particular structural elements in the upper part of the thighbone neck formed. When implants thicker in cross section are used (Figs. 2 and 3), the elements situated above the slanting part of the implant completely disappear or are made of much less dense material (light shades of grey or the white color). 


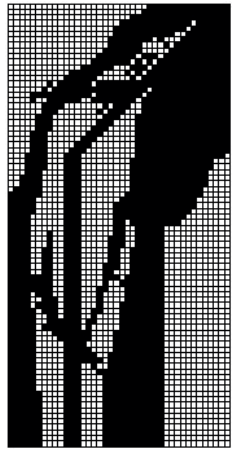

(a)

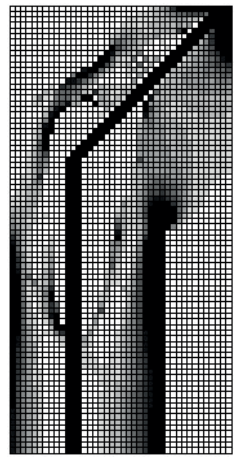

(d)

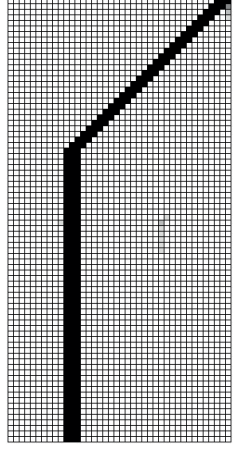

(b)

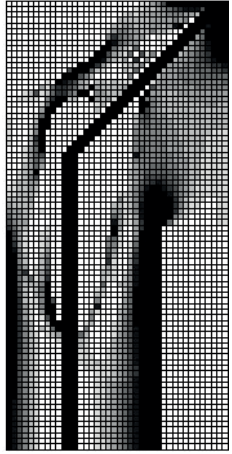

(e)

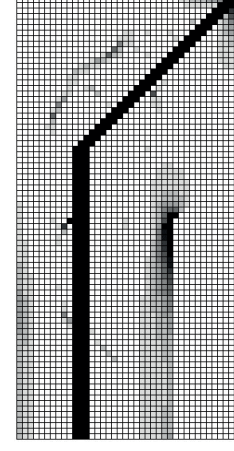

(c)

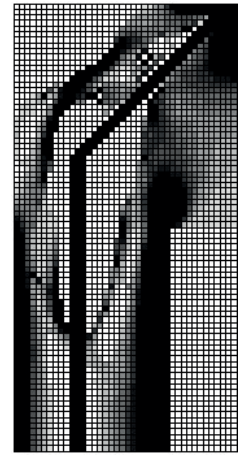

(f)
Fig. 6 Topologies of density distribution in step 7 under loading with force $P$ ("thin" implant) for $\alpha=0.50 ; 0-1$ (a); 0-1 (b); 0-0.1 (c); 0-0.02 (d); $0-0.015$ (e); 0-0.001 (f)

Neither the bone structure in the areas situated above the slanting part of the implant nor on the left side in the thighbone crown region weakens as a result of the increase in the available mass combined with the use of a reasonably thin implant. Similar correlations as at a relatively lower coefficient a are observed. It is apparent that when a thin implant with a relatively small cross section is used, also the bone tissue is engaged in carrying the load by the implant. Some of the load from the upper part of the implant head is carried by the implant and some by the bone. The spongy bone surrounding the implant does not atrophy but conjoined with the cortical bone transfers the loads to the lower parts of the thighbone.

The changes in bone structure remodeling after implantation become even more visible when the model is loaded with force W. Fig. 7 shows an exemplary solution for loading with force $\mathrm{W}$, which was obtained for the same control parameters as the topology for loading with force $\mathrm{P}$, shown earlier in Fig. 4.

A similar comparison was made for a "thin" implant. A solution for loading with force $\mathrm{P}$ and a solution for loading with force W are shown in respectively Fig. 6 and Fig. 8.

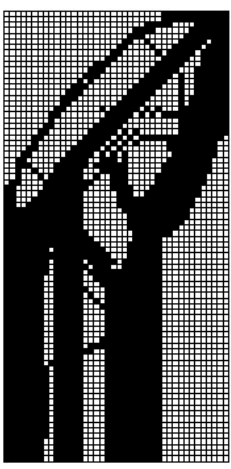

(a)

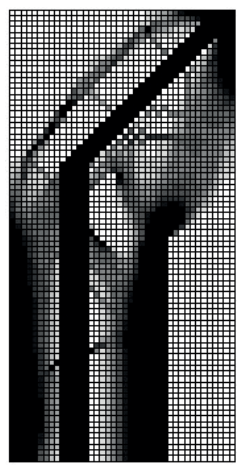

(d)

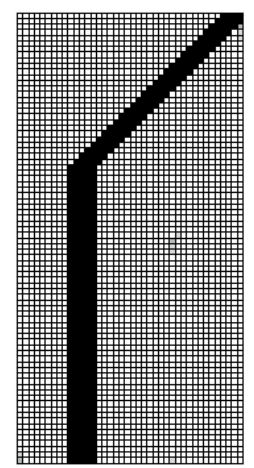

(b)

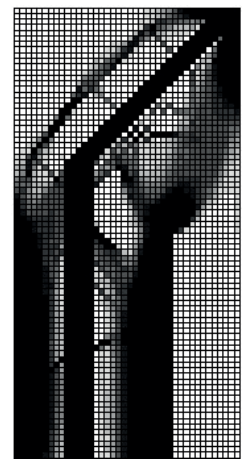

(e)

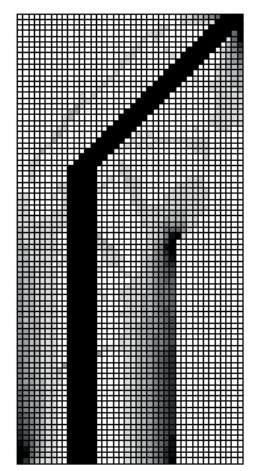

(c)

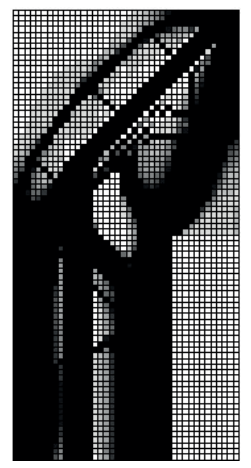

(f)
Fig. 7 Topologies of density distribution in step 15 under loading with force $W$ for $\alpha=0.50$; 0-1 (a); 0-1 (b); 0-0.1 (c); 0-0.02 (d); 0-0.015 (e); $0-0.001$ (f)

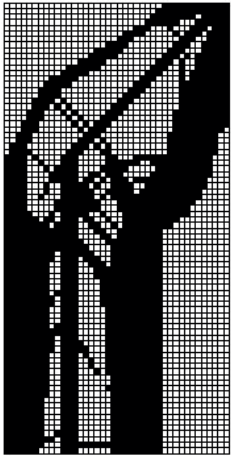

(a)

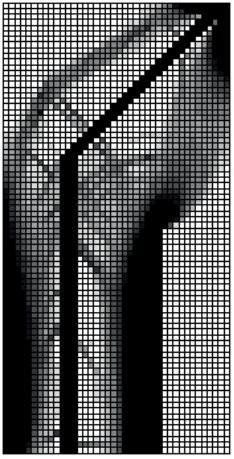

(d)

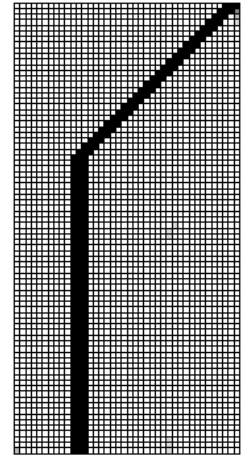

(b)

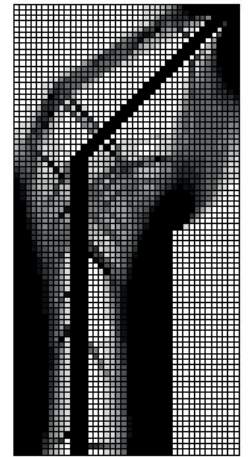

(e)

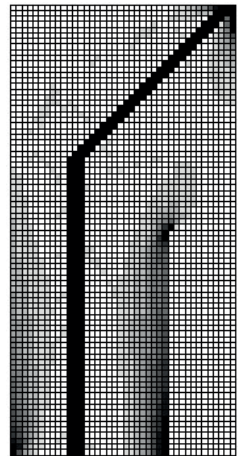

(c)

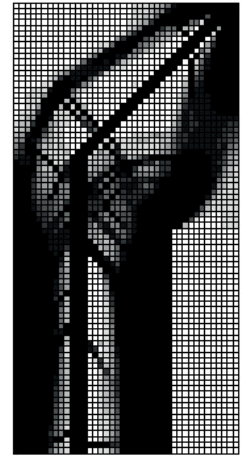

(f)
Fig. 8 Topologies of density distribution in step 20 under loading with force $W$ ("thin" implant) for $\alpha=0.50 ; 0-1$ (a); 0-1 (b); 0-0.1 (c); 0-0.02 (d); $0-0.015$ (e); 0-0.001 (f) 
It is apparent that the bone structure in the distribution shown in Fig. 8 (force $W$ ) differs from the topology in Fig. 6 (force $P$ ). In the solution for load $\mathrm{W}$ one can discern more thinner bars which join the implant. An examination of the solutions (Fig. 7 or 8 ) reveals that the single thin bars model spongy bone with a trabecular structure. The bars surround the implant forming with the latter a network of connections, which means that the bone tissue and the implant form a union and began to interact. Relatively more thin bars, resembling spongy bone with a trabecular structure, join the "thin" implant (Fig. 8(f)). In the case of the implant with a slightly larger cross section, the number of such members decreases (Fig. 7(f)).

In the solutions with force W (Fig. 7 or 8 ) there is much less material on the right side of the thighbone head than in the distributions for loading with force $P$, i.e. material in this case (force $W$ ) is distributed in the external cortical bone on the left and right side. The external edge of the thighbone head is connected via the thin bars with the implant. Numerous bars also originate from the cortical bone and extend towards the implant.

Implantation disturbs the structure of the bone, regardless of the cross section of the implant. At the contact between the different materials (different densities) adverse phenomena occur, contributing to the degradation of the osseous system (the atrophy of structural bone elements in certain areas). It should be noted that the changes in the bone structure resulting from the introduction of the implant are similar to the ones observed in actual long-term post-implantation studies reported in the literature [11].

It appears from the presented distributions that the bone structure is differently remodeled depending on the adopted loading configuration. Bone is continually subjected to different loads and in each of the situations it must be possibly strongest.

The density distribution of the bone tissue into which an implant has been introduced differs from that of the bone tissue without the implant. For example, a comparison of the solution (without implant) shown in Fig. 4(a) with the one (with a "thick" implant) in Fig. 4(b) reveals a significant change in the bone structure. The elements forming the upper part of the thighbone head completely disappear as a result of the implantation. No such phenomenon, consisting in the elimination of material from the area above the slanting part of the prosthesis, is observed when a "thinner" implant is employed (Fig. 6). In this case, the bone interacts with the implant: some of the load is carried by the implant and some via the spongy bone is transferred outside to the cortical bone. It is apparent that if the bone is relieved too much (whereby a substantial part of the load is carried by the implant alone), the bone will atrophy.

Significant changes in material distribution also become apparent for force $W$. The outline of the thighbone is similar, but in the solutions with the implant (Figs. 7 and 8) thin bars have formed. Such structures are not observed in the thighbone without the implant.

Summing up this section, one can say that that the material distributions show where the bone material should be located in an optimal distribution. Let us repeat that in the human body during its life the bone tissue is being degraded and subsequently remodeled in such places and in such a shape and assuming such a structure as the one emerging from the analysis of its distribution optimal with regard to the loading state of the bone structure. Let us repeat that in the case of implantation, it is the implant which carries most of the load. Then from the optimal bone distribution point of view no bone is needed in the neighborhood of the implant and so the human body does not remodel the bone in the neighborhood of the implant. This is what actually happens. The optimal distributions of bone material around the implant, obtained on the basis of structure compliance minimization, confirm precisely the fact that no bone is needed in the neighborhood of the implant. Therefore it can be concluded that as the load is carried by the implant, then the human body simply does not remodel the bone in the neighborhood of the implant and consequently after some time since the implantation the bone around the implant may atrophy or atrophies.

\subsection{Analysis of thinner implant cross section and reduced implant density (force $\boldsymbol{W}$ )}

Here material distributions for different implant densities $(1.0,0.9,0.8,0.7,0.6,0.5,0.4,0.3)$ instead of the stiff implant's density of 1 were studied. Computations were performed for the "thin" implant. The design area was loaded with force $W$ and mass reduction coefficient was adopted.

The stiffness of a member in compression or tension is usually defined as a product of cross-sectional area A and Young's modulus E. The above implant densities lower than unity should be understood as implant stiffnesses (EA) appropriately reduced by reducing the implant's E-modulus.

The aim of the computations was to find out what the bone material distributions would be for a relatively weaker implant. It was shown above that when a too stiff (too large in cross section) implant is inserted, the bone surrounding it undergoes degradation, which results in 
adhesion loss (implant loosening). In the optimization process no material is distributed around a too stiff implant. It could be expected that if the implant was weakened to some extent, then as a result of optimization numerous connections with the bone would form around the implant. It this proved to be the case, the implication would be that implants should be less stiff and material specifications should be more similar to those of the bone.

If in the computations presented below an implant with a density of, e.g., 0.4 is mentioned, this means that this implant density value remains the same throughout the optimization process while the available mass is subject to optimal distribution. The results of the computations are presented in the Tables 1-3.

Table 1 shows the topologies obtained for different implant densities. These are solutions of the material-void type. It is clearly apparent that the lower the implant density, the wider the thighbone's cortical bone, i.e. the vertical bone elements located in the thighbone's lower part are wider and more interconnected. This is particularly visible if one compares the topology with the 1.0 density implant with the topology with the 0.3 density implant.
A comparison of the solutions presented in table 1 shows that the solutions at lower implant density are closer to the solution without the implant .

Table 2 shows density distribution under the same scheme as above (e.g. Fig. 7 or 8), but on suitable density scales. This time the topologies are shown on the scales of $0-0.03,0-0.01$ and $0-0.05$, whereby one can clearly see how the material distributions change depending on the implant material density. For instance, if at the same density scale of $0-0.03$ one compares the extreme solutions (implant densities of 1.0 and 0.3 ), it becomes clear that in the case of the weaker implant the bones are markedly stronger (filled with material of higher density). The same is observed if the other two columns of the obtained topologies are examined in the same way as above. Examining, going from top to bottom, each of the columns separately one can see how bone density smoothly changes.

When one examines Table 3, one can notice that the topologies presented here, regardless of implant density, are in a lighter shade of grey than the ones shown in Table 2. The weaker the implant, the blacker the topologies become, which means that bone material gets concentrated

Table 1 Topologies with implant, shown on scale of 0-1

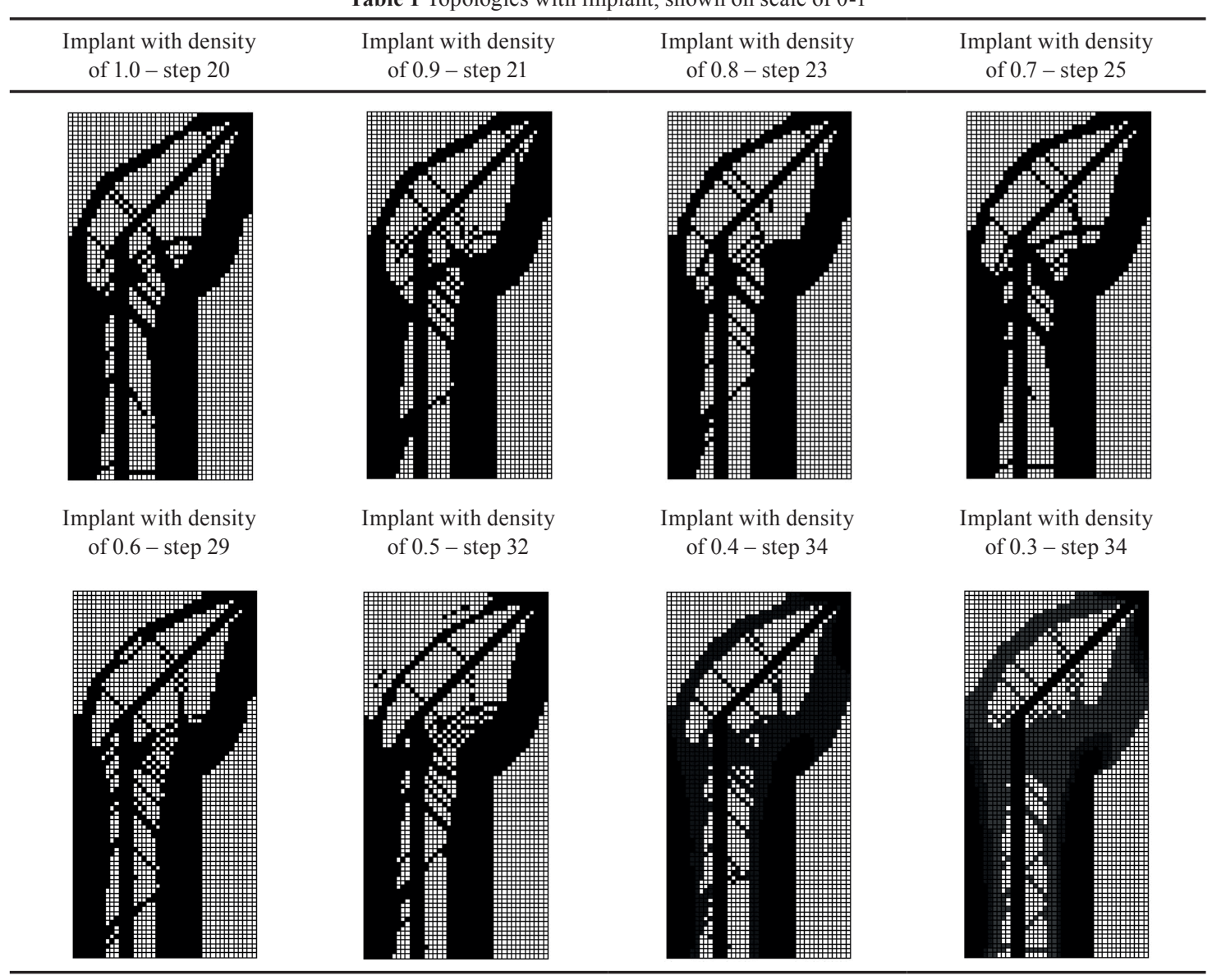


Table 2 Topologies with implant, on three selected scales

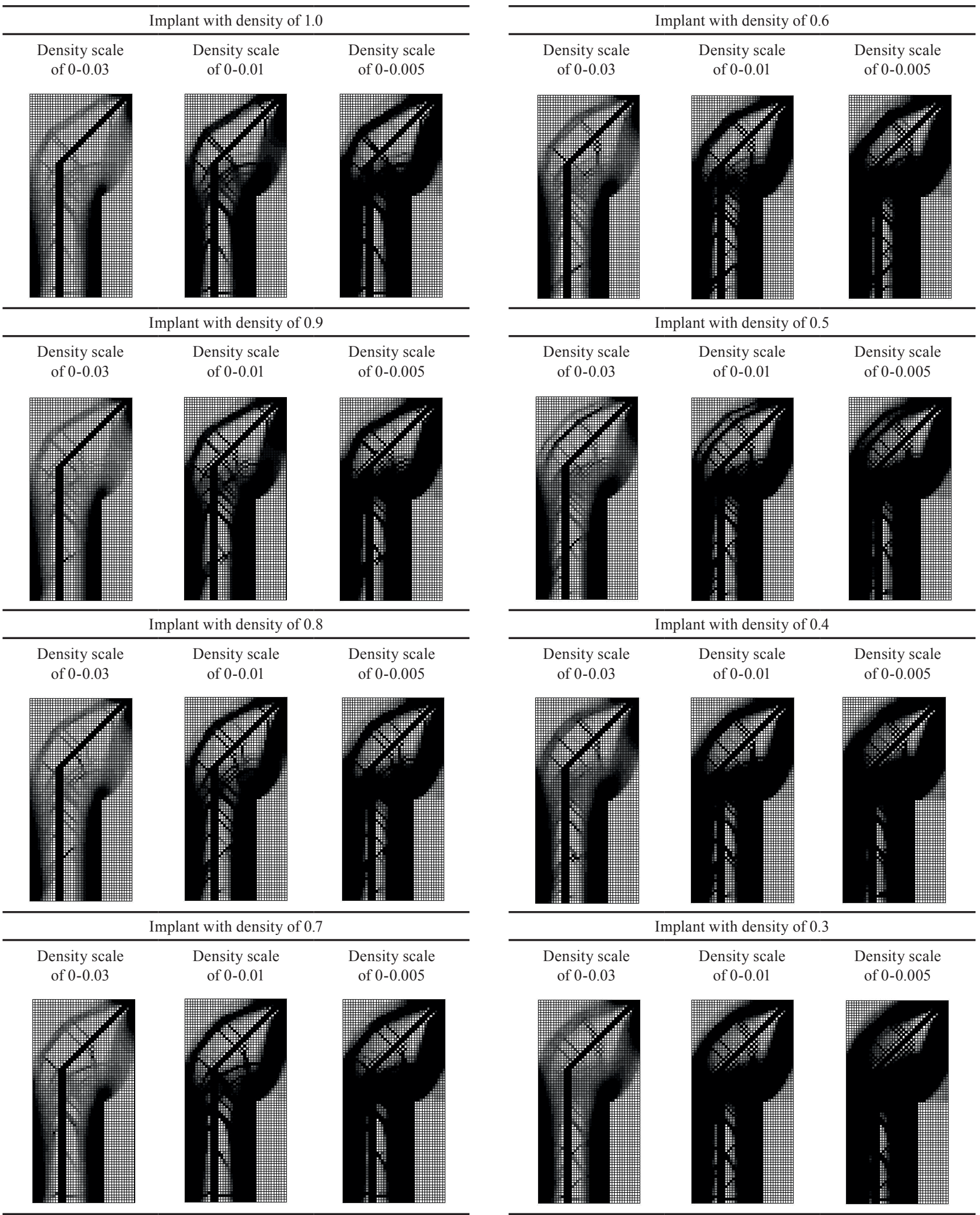


Table 3 Topologies without implant, on four selected scales

Density distribution
on scale of 0 - 1

in certain places. The aim of this concentration is to ensure interaction between the implant and the bone. In the case of the bone without the implant, one obtains a more uniform distribution of relatively weak material. Let us examine the distributions on the scale of 0-0.005. Let us compare the topology (the implant with a density of 1.0) shown in Table 2 with the one in Table 3. If an implant is introduced into the bone, connections with the implant form and because of the latter's relatively high stiffness also the connections must be stiffer, whereby material is being concentrated on the connections with the implant. This is visible in the topologies: the topology in Table 3 is fuzzier than the one in Table 2, where the material is more concentrated. The introduction of a weak implant (with a density of 0.3 ) results in the formation of many connections between the bone and the implant. Thus, it can be concluded that a weaker implant stimulates bone structure remodeling.

\section{Conclusions}

As part of this research, numerical analyses of the model of the thighbone with and without an implant have been carried out. The fact, known from clinical studies, that

\section{References}

[1] Kutyłowski, R., Szwechłowicz, M. "Topology Optimization - a Variational Formulation of the Problem and Example Application", Periodica Polytechnica Civil Engineering, 64(1), pp.101-121, 2020. https://doi.org/10.3311/PPci.13999

[2] Kutyłowski, R., Szwechłowicz, M. "Application of topology optimization to thighbone and thighbone/implant structure modelling", Archives of Civil and Mechanical Engineering, 19(4), pp. 1006$1019,2019$.

https://doi.org/10.1016/j.acme.2019.05.007 the bone in the vicinity of the implant undergoes degradation has been numerically confirmed. This was shown using as an example a model of the endoprosthesis inserted into the thighbone. From an analysis of normalized deformation energy values one can obtain maps showing the strain of the particular structural components and so indicating what mechanical properties should characterize a given structural component of the implant or the bone. It has been numerically proved that the implant should be made of a material having similar mechanical properties as the bone. Then no degradation of the bone around the implant occurs and, moreover, numerous connections between the bone and the implant form, as shown above. Thus, by using proper materials to produce implants one can stimulate the remodeling of bone tissue in a way ensuring the most complete embedding of the implant and so its long and safe service. The above conclusions are confirmed in [3], where it is proved that a graded cellular implant can minimize concurrently bone resorption and implant interface failure.

[3] Khanoki, S. A., Pasini, D. "Multiscale Design and Multiobjective Optimization of Orthopedic Hip Implants with Functionally Graded Cellular Material", Journal of Biomechanical Engineering, 134(3), Article number: 031004, 2012. https://doi.org/10.1115/1.4006115

[4] Karachalio, T. (ed.) "Bone-Implant Interface in Orphopedic Surgery", Springer, London, UK, 2014. https://doi.org/10.1007/978-1-4471-5409-9 
[5] Ayatollahi, M. R., Mirmohammadi, S. A., Shirazi, H. A. "The tension-shear fracture behavior of polymeric bone cement modified with hydroxyapatite nano-particles", Archives of Civil and Mechanical Engineering, 18(1), pp. 50-59, 2018.

https://doi.org/10.1016/j.acme.2017.06.001

[6] Kerner, J., Huiskes, R., van Lenthe, G. H., Weinans, H., van Rietbergen, B., Engh, C. A., Amis, A. A. "Correlation between pre-operative periprosthetic bone density and post-operative bone loss in THA can be explained by strain-adaptive remodeling", Journal of Biomechanics, 32(7), pp. 695-703, 1999. https://doi.org/10.1016/S0021-9290(99)00041-X

[7] Boyle, C., Kim, I. Y. "Comparison of different hip prosthesis shapes considering micro-level bone remodeling and stress-shielding criteria using three-dimensional design space topology optimization", Journal of Biomechanics, 44(9), pp. 1722-1728, 2011. https://doi.org/10.1016/j.jbiomech.2011.03.038

[8] Belcarz, A., Ginalska, G., Ślósarczyk, A., Paszkiewicz, Z. "Kompozyt bioaktywny oraz sposób wytwarzania kompozytu bioaktywnego" (A bioactive composite and a method of producing the bioactive composite), Patent Office of the Republic of Poland, Warsaw, Poland, Patent number: 387872, 2009. (in Polish)

[9] Yamako, G., Janssen, D., Hanada, S., Anijs, T., Ochiai, K., Totoribe, K., Chosa, E., Verdonschot, N. "Improving stress shielding following total hip arthroplasty by using a femoral stem made of $\mathrm{b}$ type Ti-33.6Nb-4Sn with a Young's modulus gradation", Journal of Biomechanics, 63, pp. 135-143, 2017. https://doi.org/10.1016/j.jbiomech.2017.08.017
[10] Telega, J. J., Lekszycki, T. "Bone remodelling as a time-dependent elastic shape optimization problem", Acta of Bioengineering and Biomechanics 3, Supplement 2, pp. 555-562, 2001.

[11] Podrez-Radziszewska, M., Haimann, K., Dudziński, W., Ozonek, W. "Uszkodzenia endoprotezy stawu biodrowego jako przyczyna niepowodzen alloplastyki" (Damage to the hip joint endoprosthesis as the cause of alloplasty failures), Chirurgia Narządów Ruchu i Ortopedia Polska, 68, pp. 169-172, 2003. (in Polish)

[12] Khoda, A. K. M. B., Ozbolat, I. T., Koc, B. "Engineered Tissue Scaffolds With Variational Porous Architecture", Journal of Biomechanical Engineering, 133(1), Article number: 011001, 2011. https://oi.org/10.1115/1.4002933

[13] Hardisty, M. R., Zauel, R., Stover, S. M., Fyhrie, D. P. "The Importance of Intrinsic Damage Properties to Bone Fragility: A Finite Element Study", Journal of Biomechanical Engineering, 135(1), Article number: 011004, 2013. https://doi.org/10.1115/1.4023090

[14] Ridzwan, M. I. Z., Shuib, S., Hassan, A. Y., Shokri, A. A., Mohamad Ibrahim, M. N. "Problem of Stress Shielding and Improvement to the Hip Implant Designs: A Review", Journal of Medical Sciences, 7(3), pp. 460-467, 2007. https://doi.org/10.3923/jms.2007.460.467

[15] Sumner, D. R. "Long-term implant fixation and stress-shielding in total hip replacement", Journal of Biomechanics, 48(5), pp. 797800, 2015.

https://doi.org/10.1016/j.jbiomech.2014.12.021 